\title{
Impact of implementation of auxiliary bias-windings on controllable inductors for power electronic converters
}

\author{
Jonas Pfeiffer (D, Pierre Küster (D), Yeliz Erenler, Ziyad H. S. Qashlan and Peter Zacharias \\ UNIVERSITY OF KASSEL \\ Department of Electrical Power Engineering (EVS) \\ Wilhelmshöher Allee 71 \\ 34121 Kassel, Germany \\ Tel.: +49 / (0)561 - 8046344 \\ Fax: +49 / (0)561 - 8046521 \\ E-Mail: jonas.pfeiffer@uni-kassel.de \\ URL: http://www.uni-kassel.de/eecs/evs
}

\section{Acknowledgements}

Parts of the work presented in this paper have been supported by the German Federal Ministry of Education and Research (BMBF). Project funding reference number:16EMO0234. Responsibility for the contents of this publications lies with the authors.

The authors thank the SUMIDA Components \& Modules GmbH in Obernzell, Germany, for manufacturing or machining most of the ferrite cores which were used to generate the presented measurement results.

\section{Keywords}

«Device characterization», «Device modeling», «Magnetic device», «Hardware (not only Software)», «Passive component»

\begin{abstract}
Magnetic devices are an important part of electronic converters. They can be used to control some properties of the converters. There are three different control methods using an auxiliary bias-winding. In the presented paper, the impact of the implementation of the auxiliary bias-winding was studied for mixed biasing. Seven different designs were investigated in small signal measurements and 3D-FEMsimulations. The results were proven by calculated results of equivalent diagram circuits, which have been developed. Regarding the standard crosswise current arrangement all designs show the same results. In a horizontal aux. current arrangement, the effect of the VAG technique is mitigated. Investigations of a vertical aux. current arrangement results in an unsymmetrical magnetization curve.
\end{abstract}

\section{Introduction}

An important focus of every power electronic circuit regarding controllability and loss reduction still lies on semiconductor devices. In consequence of wide band gap technologies, it becomes possible to increase switching frequency by simultaneously decreasing switching and conduction losses. Before using wide band gap semiconductor devices in an application several investigations relating to overload and thermal conditions as well as error management are essential.

In contrast, magnetic devices are characterized by a significant higher robustness and a higher reliability regarding thermal stresses or cosmic radiation. However, in power electronic applications magnetic components typically are the devices which occupy a significant portion of the overall volume and weight. Furthermore, they tend to cause the highest component costs. Especially in mobile applications, controllable magnetics can be used to afford size and cost reduction. This paper is 
focused on current controlled magnetic devices. Ferrite materials are used for simulations and verification measurements.

\section{Motivation and application approaches}

The motivation of this paper is to get a deeper understanding of the usage of controllable magnetic devices. With their usage, an additional degree of freedom is gained over the course of the application's design process.

Current controlled magnetic devices can be used e.g. for size and cost reduction as it is shown in [1]. Especially in automotive applications, a significant size and weight reduction is essential. Further uses are possible, especially in resonant topologies, to improve the overall efficiency by decreasing the resonant circuit's frequency range and its reactive power losses.

Investigations and discussions of applications for controllable inductors in power electronic converters are presented in [2].

\section{Systematization}

A magnetic device gets adjustable by flux interactions of differently aligned magnetic fields. Flux interactions can be achieved by using permanent magnets. Their usage in inductors for power electronic converters is investigated and discussed in [3].

A feasible way to adjust and even control magnetic devices is to use auxiliary windings which are wound on or introduced into the core material. A current, which flows through the auxiliary winding, leads to an additional magnetic flux which saturates parts of the core and effect its overall reluctance. There are three different methods of superimposing magnetic fields. They are shown in Fig. 1.

Reference [2] gives an overview of the three different methods of basic control approaches and shows possible applications.

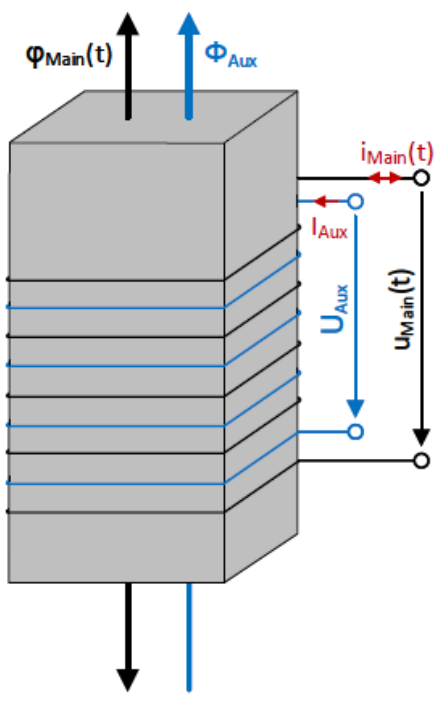

a)

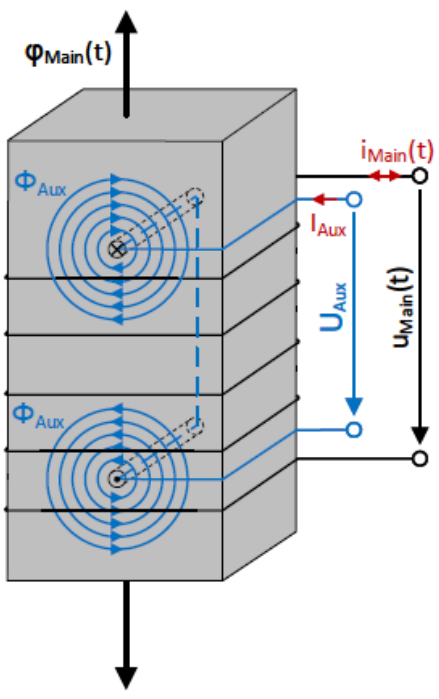

b)

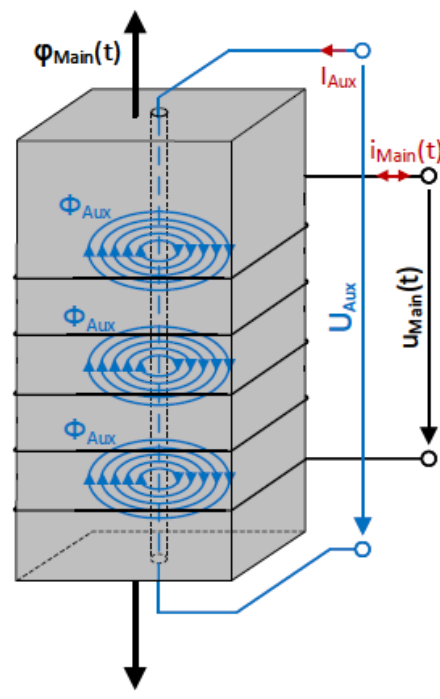

c)

Fig. 1: Three different methods of superimposing magnetic fields by using an additional winding wound on or introduced into the core material. a): Parallel biasing; b): Mixed biasing; c): Orthogonal biasing [2]

For the following investigations, the method of mixed biasing in the form of the virtual air gap (VAG) technique is used. In this technique the auxiliary winding is introduced into the magnetic core through drill holes which are orthogonal aligned to the main flux. The auxiliary bias current occurs an auxiliary magnetic field. This field generates an additional auxiliary magnetic flux which is either parallel or orthogonal to the main flux. A detailed analysis and discussion of a virtual air gap variable reactor (VAG-VR) is presented in [4]. References [5] - [11] give additional information about the fundamental functionality of the VAG technique. 


\section{Manufacturing and machining process}

Such as ceramics, ferrite is a very fragile and brittle material. If machined wrong, the magnetic core will be damaged or even breaks and becomes useless. A suitable procedure to drill the required holes into a magnetic core is the usage of an Ultrasonic/Sonic Driller/Corer (USDC). To prevent any damage to the drilling tool, it is important to comply with the manufacturing limits and tolerances of the UDSC, regarding its drilling depth in conjunction with the hole diameter.

If high quantities are necessary, e.g. in case of an industrial series production, the required holes can be integrated into the press dies.

\section{Impact of implementation of auxiliary bias windings}

Depending on the magnetic device's characteristics, high bias currents are needed in the auxiliary winding to saturate the area around the drill holes. In this situation, a suitable approach is to increase the number of turns of the auxiliary winding to decrease the needed bias current:

With:

$$
\Phi=\frac{\mathrm{F}}{R_{m, \text { total }}}=\frac{N * I}{R_{m, \text { total }}}
$$

- $\Phi$ : Magnetic flux

- F: Magnetomotive force

- $R_{m, t o t a l}$ : Total magnetic reluctance

- $\quad N$ : Number of turns

- $\quad I$ : Electric current

According to (1), the bias current can be decreased by the factor the number of turns is increased to generate a similar magnetic flux. A lower auxiliary current positively impacts the efficiency as well as the volume of the auxiliary current-source circuit.

There are various possibilities to implement an auxiliary bias-winding with multiple turns. To examine any impact of the auxiliary winding's arrangement, three different designs shown in Fig. 2 were investigated. The designs vary in the return path of the auxiliary bias-winding.

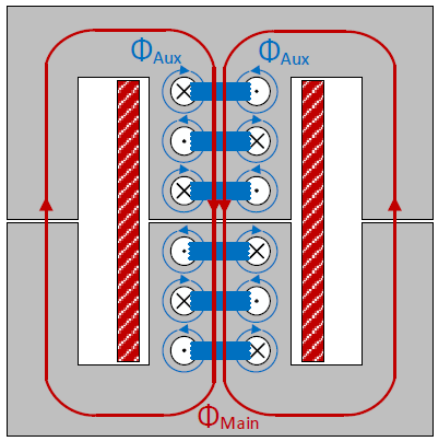

Design A

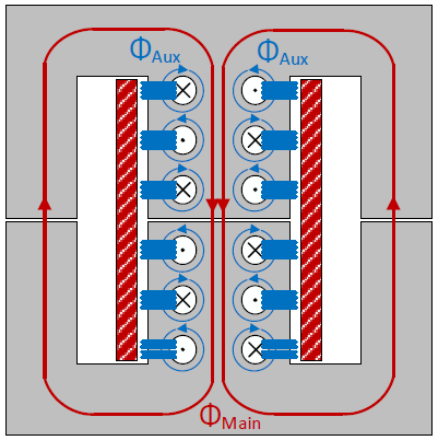

Design B

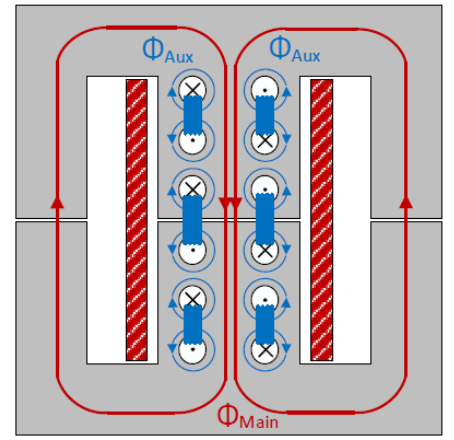

Design C

Fig. 2: Various designs of implementation of an auxiliary bias-windings. The main winding (red) encloses the auxiliary windings (blue) with multiple turns.

An E55/28/21 core made of "N87 material" (EPCOS/TDK) was used to realize the devices. The main winding consists of ten turns of litz-wire. Furthermore, an overall air gap of $0,14 \mathrm{~mm}$ was implemented.

For all three designs measurements started with one turn of the auxiliary bias-winding. The number of turns was increased to two turns and again up to four turns. By increasing the number of turns, the auxiliary bias-current was decreased according to (1) to keep the magnetomotive force even, whereby a comparison of the bias points is ensured.

All shown experimental results are small signal measurements by using a Wayne Kerr Precision Magnetics Analyzer 3260B. The analyzer measures the magnetic device's differential inductivity by increasing the main current from $1 \mathrm{~A}$ to $30 \mathrm{~A}$ in steps of $1 \mathrm{~A}$. The measurement results have been 
numerically integrated to show the magnetic flux linkage as a function of the magnetomotive force of the main winding. Between the measurement sweeps, the auxiliary current was increased gradually from $0 \mathrm{~A}$ to $100 \mathrm{~A}$ in steps of $10 \mathrm{~A}$.

In a first step, it was investigated if the increasing number of turns of the auxiliary bias-winding within a defined design has any impact on the behavior of the magnetic device.

Figure 3 a) shows the experimental results of Design B for one, two and four turns of the auxiliary bias-winding. The different graphs of the same auxiliary magnetomotive force show a comparable course. The other investigated designs $\mathrm{A}$ and $\mathrm{C}$ show similar results. Therefore, it must be concluded that, according to (1), the number of turns of the auxiliary bias-winding within a defined design has no impact on the behavior of the magnetic device.

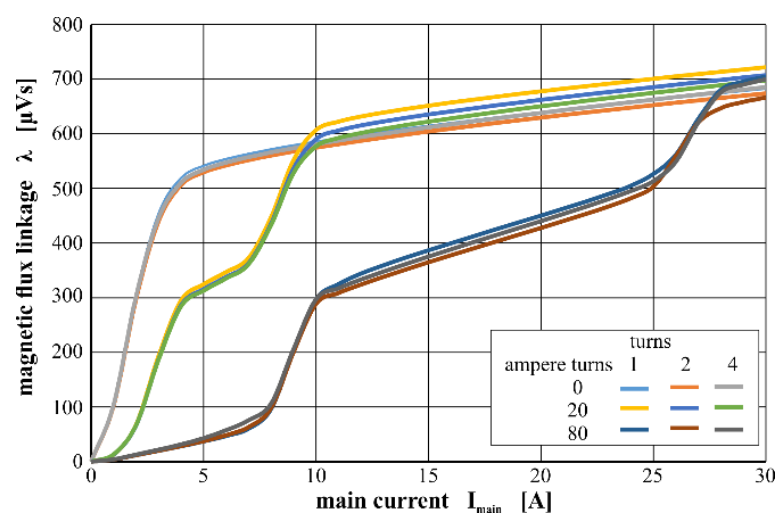

a)

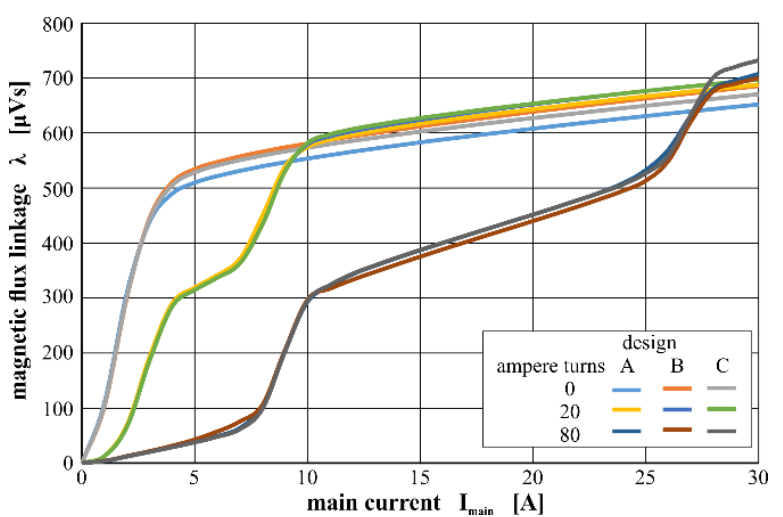

b)

Fig. 3: Experimental results

a): Comparison of Design B with different number of turns of the auxiliary bias-winding;

b): Comparison of Design A, B and C with four turns of the auxiliary bias-winding

In a second step, it was investigated if the implementation of the auxiliary bias-winding has any impact on the behavior of the magnetic device. Therefore, the results of the three investigated designs were compared for one, two and four turns of the auxiliary bias-winding.

The comparison of the three designs for four turns is shown in Fig. 3 b). Again, the courses of the graphs with the same auxiliary magnetomotive force are comparable. The comparison of the results for one and two turns of the auxiliary bias-winding are similar. The experimental results lead to the conclusion that the arrangement of the auxiliary bias-winding has no impact on the magnetic device's behavior.

On closer inspection, multiple risings of the courses in Fig. 3 a) and b) are notable. The reasons for that effect is not completely clear. A possible explanation are flux interactions between the resulting different saturated zones around the drill holes and the main magnetic flux.

For this purpose, the effects of individual nonlinear magnetized sections were investigated using simplified equivalent circuit diagrams. Fig. 4 shows the corresponding procedure.

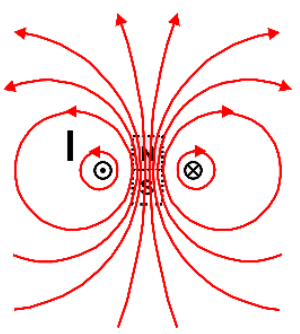

a)

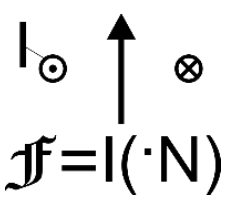

b)

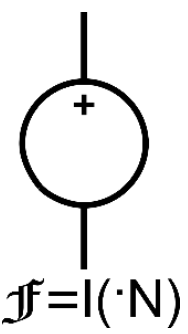

c)

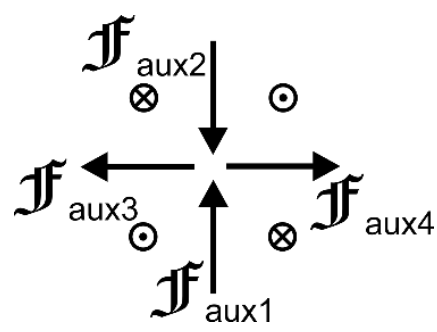

d)

Fig. 4: Development of the equivalent circuit diagram a): Magnetic field of a conductor pair; b): Its magnetomotive force effect; c): Equivalent circuit diagram; d): Interaction of several conductor pairs 
The magnetic flux is driven by the electromagnetic forces in a network of non-linear magnetic resistances. By superposition with the main magnetic field, the characteristic magnetization curve $\lambda$ (I_main) is obtained. As a simple application example, Fig. 5 a) shows the structure of a model for design variant D. Fig. 5 b) shows the simulation results obtained, which reflect the behavior of the magnetization characteristic curve qualitatively, but also in part quantitatively.

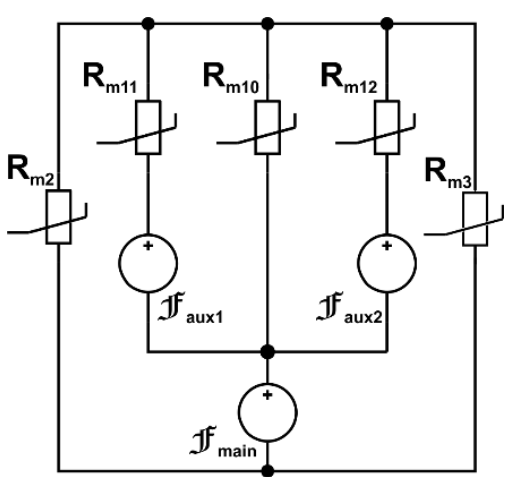

a)

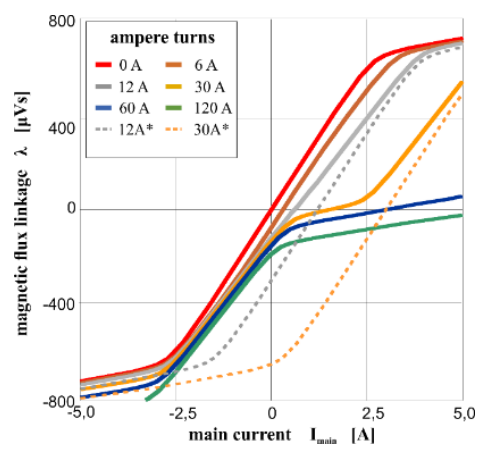

b)

Fig. 5: Rough reluctance model for the winding construction according to design $D$ with vertical auxiliary flux.

a): Construction of the model with similarly directed field of the premagnetization;

b): Characteristic curves determined by simulation based on the reluctance network, (dotted lines: same premagnetization without using the holes)

In Fig. 5 b) you can also see for comparison the effect of a simple winding under the main winding with the same premagnetization as a dotted line. One can clearly see the different effects on the symmetry of the magnetization curve. These could also be proven by measurement (see below).

\section{Impact of arrangement of the auxiliary current}

When using mixed biasing, the directions of the auxiliary current in adjacent drill holes are opposite aligned what results in a crosswise arrangement as it is shown in in Fig. 2. To get a deeper understanding of the impact of the auxiliary bias-winding on the magnetic device, the arrangement of the current direction was investigated.

In a first step, a horizontal current arrangement was applied by using a variation of the designs B and C. The directions of the auxiliary current are no longer crosswise arranged. Instead, the auxiliary current of horizontally adjacent drill holes is same aligned. The auxiliary current in vertically adjacent drill holes remains opposite aligned. Figure 6 shows the vertical current arrangement for the new designs $\mathrm{D}$ and $\mathrm{E}$.

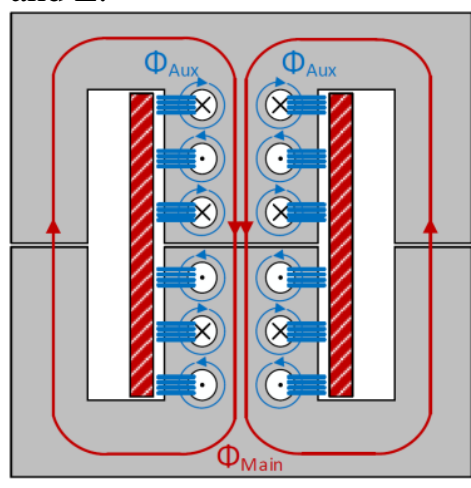

Design D

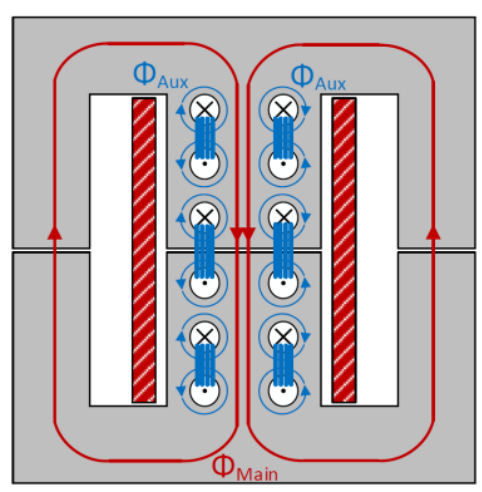

Design $\mathbf{E}$

Fig. 4: Horizontal arrangement of the auxiliary current for two designs

The experimental results, which are shown in Fig. 7, verify the simulation results of the adjusted Design E. As in a standard current arrangement the increase of the number of turns of the auxiliary 
bias-winding has no impact on the magnetic device's behavior. According to simulation results, the principle effect of mixed biasing still exists in a horizontal current arrangement and is similar to a standard crosswise arrangement.

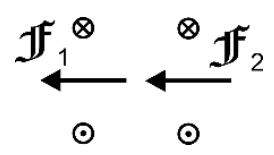

a)

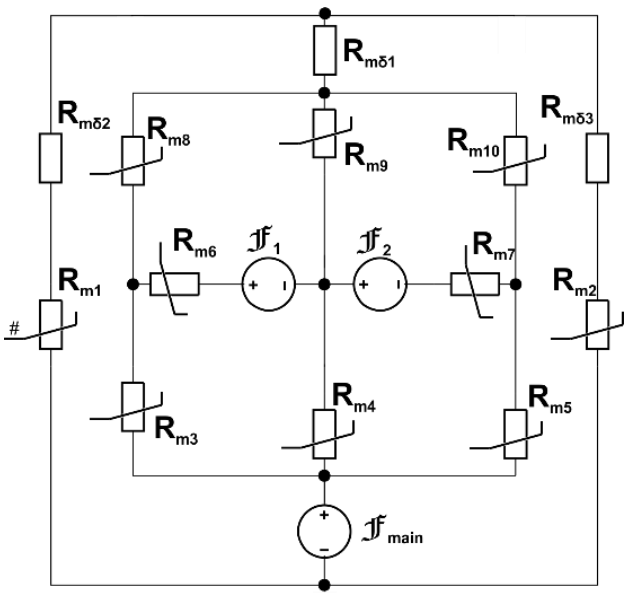

b)

Fig. 7: Simplified model structure for a pre-magnetization field transverse to the main field. a): Orientation of the MMF, b): Model structure

Figure 8 a) shows the curves of the magnetizing winding measured for design $\mathrm{E}$ at different numbers of turns and flooding of the premagnetization. The measurements clearly show that the higher MMF of the premagnetization leads to an increasing pinch-off of the magnetization range. This causes the first threshold in the magnetization curve to drop and the course of the flux to change. These processes cannot be represented by the model in Fig. $7 \mathrm{~b}$ and require 3D field calculations.

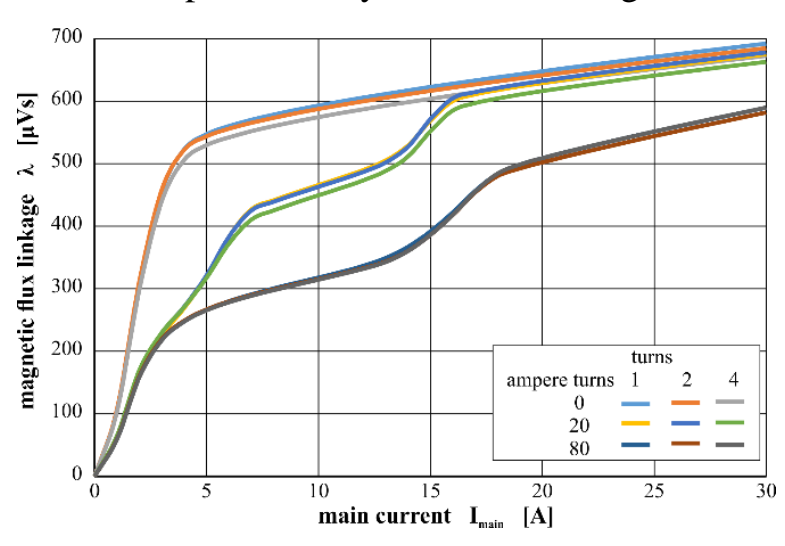

a)

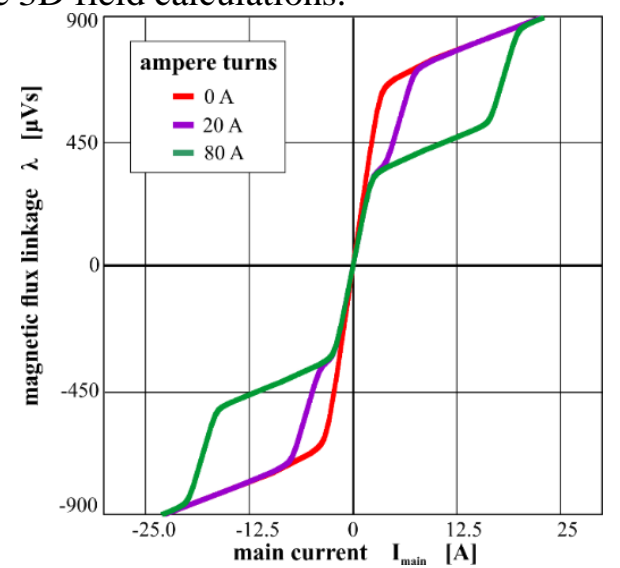

b)

Fig. 5: Experimental results of Design E (a) with different numbers of turns of the auxiliary biaswinding and calculated results with the model of Fig. 7 b)

However, compared to the results of the standard arrangement in Fig. 3, the graphs of the horizontal current arrangement in Fig. 7 show different courses. At an auxiliary magnetomotive force of $20 \mathrm{~A}$, the "plateau" is much more pronounced, what results in a "delayed" saturation at a higher main magnetomotive force. At an auxiliary magnetomotive force of $80 \mathrm{~A}$ the graphs rise immediately but their courses are less steep than in a standard arrangement, what likewise "delays" the saturation of the magnetic device.

Because of the flatter courses in Fig. 8 a), it can be said that the effect of mixed biasing gets mitigated in a horizonal current arrangement. 
In a second step, a vertical current arrangement was applied by using a variation of the designs A and B. The directions of the auxiliary current of vertically adjacent drill holes is same aligned. The auxiliary current in horizontally adjacent drill holes is opposite aligned. Figure 8 shows the vertical current arrangement for the designs $\mathrm{F}$ and $\mathrm{G}$.

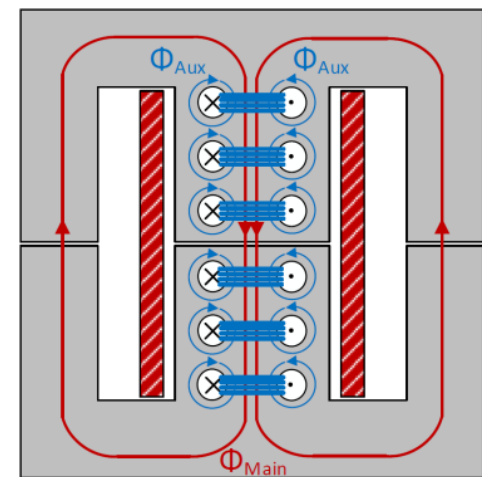

Design $\mathbf{F}$

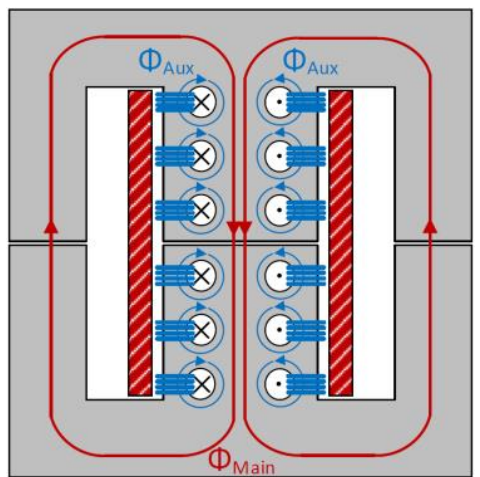

Design G

Fig. 6: Vertical arrangement of the auxiliary current for two designs

Due to the vertical arrangement the auxiliary current generates an auxiliary magnetic flux which effects the entire core geometry. Depending on the arrangement, the auxiliary magnetic flux and the main magnetic flux can be same or opposite aligned. The principle of that arrangement is similar to parallel biasing. Figure 1 a) shows the method with an auxiliary winding wound on the core instead of being introduced through drill holes. The method of parallel biasing was used in transductors (see Ref. [12]) and is still used in bias-inductors (see Ref. [13]).

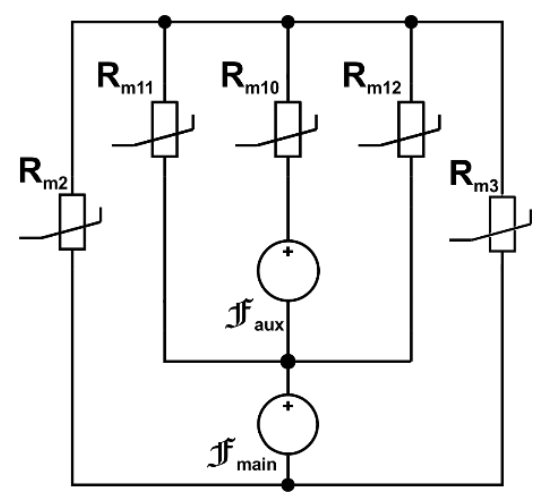

a)

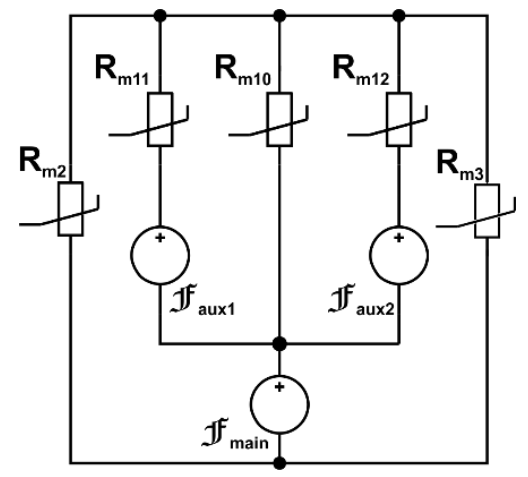

b)

Fig. 10: Simplified reluctance models to simulate the variable characteristics for the two design variants F and G. a): Design F; b): Design G

The results of the simulations with the models in Fig. 10 a) and b) are practically identical. Fig. 11 shows the comparison of practical measurements and model calculations. It should be noted that the magnetic state of space cannot be measured directly with the method used. By integrating the smallsignal measurements (see above), it is possible to determine the progressions only down to an unknown constant. Therefore, the flux value for I_main $=50 \mathrm{~A}$ for all curves was chosen to be the same as without premagnetization. In the simulation, non-linear reluctance characteristics are calculated in the same way as with resistors. Thus, the result of the superposition of different magnetic fields is also better represented. Due to the core shape and the arrangement of the holes one has an inner and an outer magnetic circuit. At higher MMFs, there is a superposition of the main field and premagnetisation in such a way that the apparent saturation of the material shifts to lower values for I_main $<50$ A. 


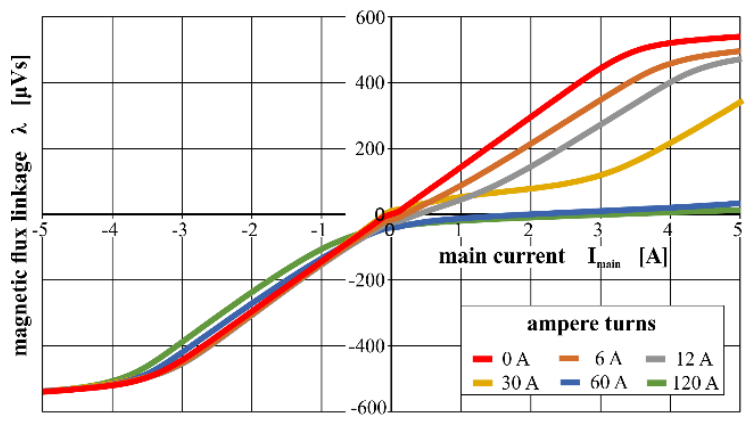

a)

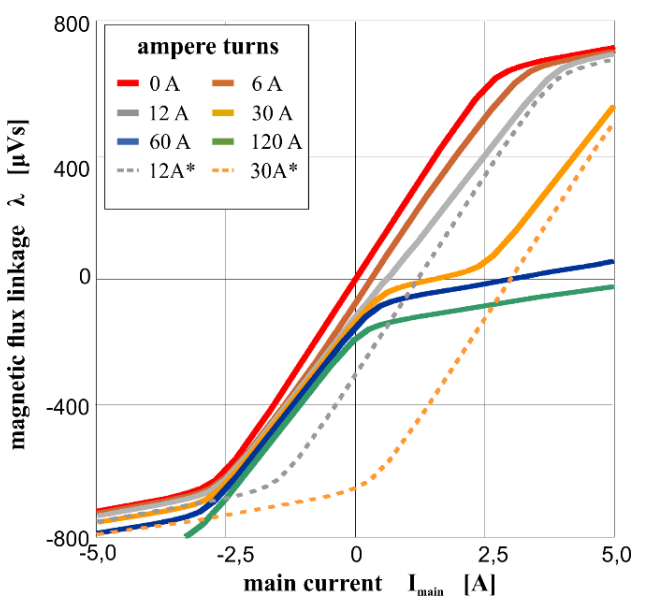

b)

Fig. 11: Results for Design F. a): Experimental results; b): Calculation results

Design F was simulated using ANSYS Maxwell. The simulation result for a main current of $5 \mathrm{~A}$ and an auxiliary magnetomotive force of 2,5 A is shown in Fig. 12.

Like the standard crosswise arrangement, the center leg of the E-core is saturated while the outer legs are less stressed. However, the flux density in the outer legs is higher in the vertical current arrangement than in the crosswise arrangement.

Regarding Fig 12 a) and b), the different behavior of the ferrite core because of the interaction with the auxiliary magnetic flux can be seen in the simulation results. Especially the outer legs show a higher flux density if both, main and auxiliary magnetic fluxes are same aligned. By increasing the main and the auxiliary current that effect leads to a saturation of the whole magnetic core as it is shown in Fig. 13.

The measurement results for Design G show similar results but twisted. The outer legs will saturate if the auxiliary magnetic flux is opposite aligned. Using a same aligned auxiliary flux, the outer legs are less effected. That also results in an unsymmetrical magnetization curve like it is shown in Fig. 11, which is rotated by $180^{\circ}$.

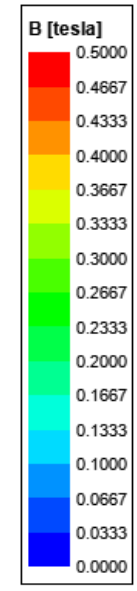

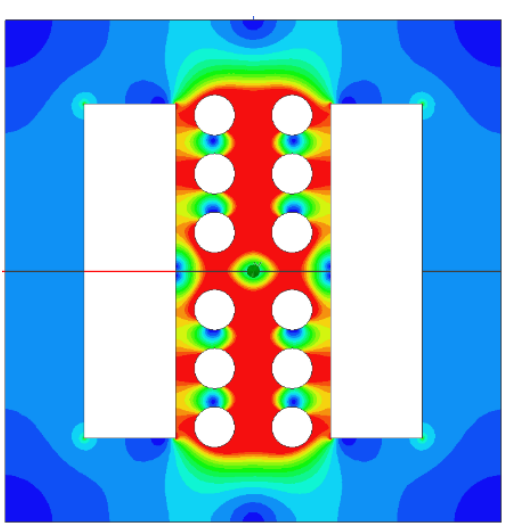

a)

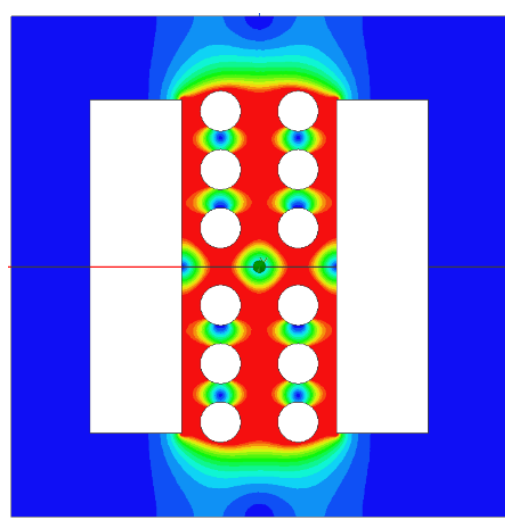

b)

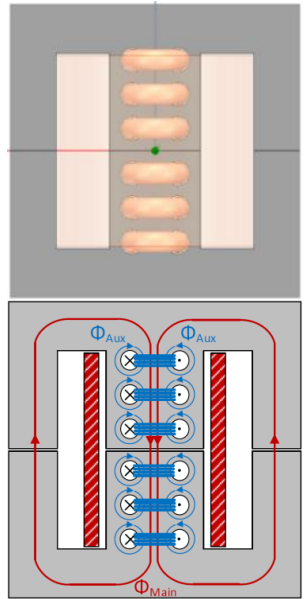

Fig. 7: Simulation results (flux density) of Design F with a main current of $5 \mathrm{~A}$ and an auxiliary magnetomotive force of 2,5 A. a): magnetic fluxes same aligned; b) magnetic fluxes opposite aligned 

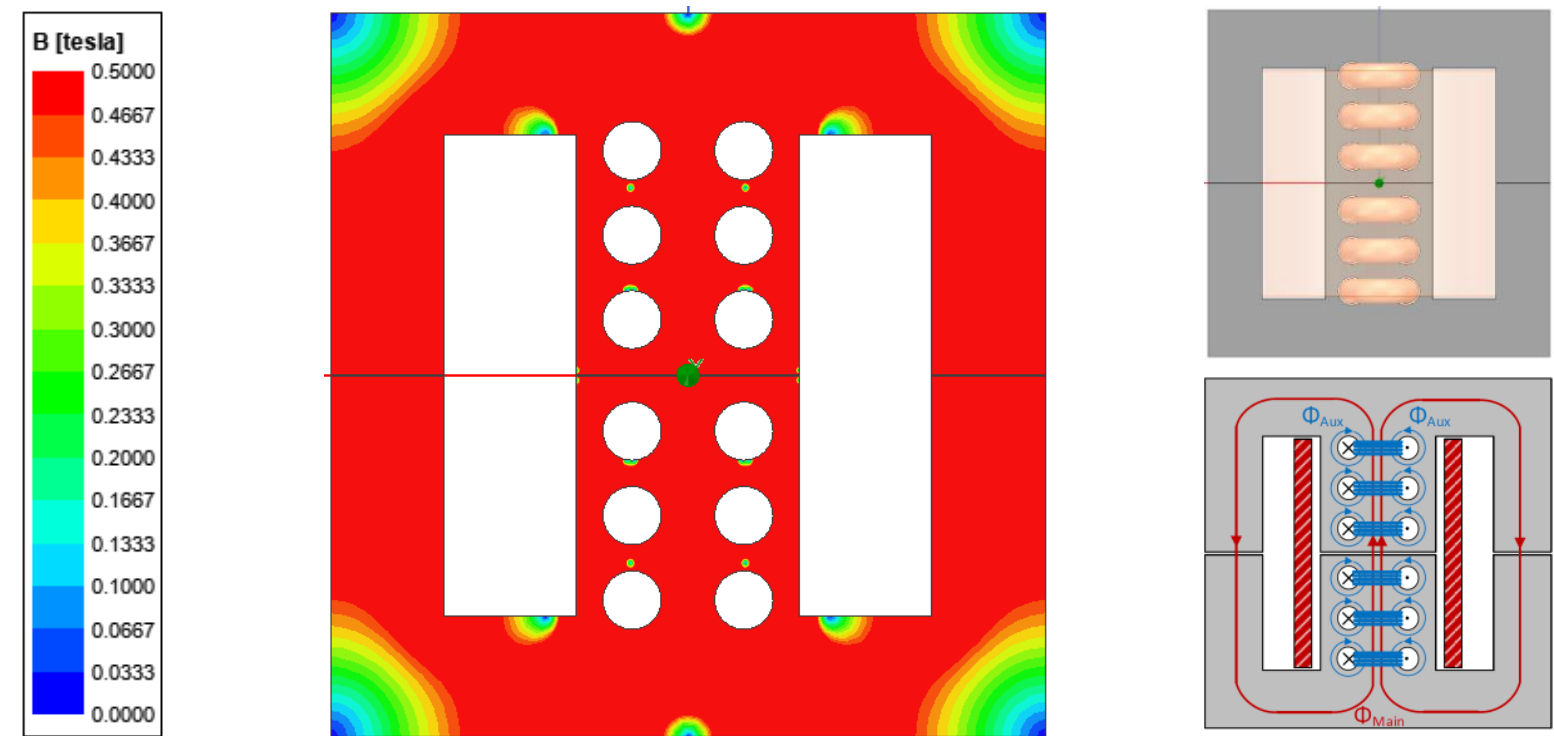

Fig. 13: Simulation results (flux density) of Design $\mathrm{F}$ with a main current of $25 \mathrm{~A}$ and an auxiliary magnetomotive force of $20 \mathrm{~A}$ (same aligned)

\section{Open research questions}

Due to the performed investigations there a several open research questions:

As it was shown there are multiple risings within the magnetization curves (e.g. Fig. 3). The exact reason for that courses is not completely clear. Furthermore, further research activities should focus the reduced saturation flux density in ferrite cores with drill holes.

In general, it should be investigated if it is possible to form the magnetization curve by using a special arrangement or diameter of the drill holes.

Beside the mentioned points, there are further open research questions which should be considered: One example is the impact of the auxiliary bias-winding on the magnetic core losses. Another point is the thermal behavior of a controllable magnetic device by using an auxiliary bias-winding. A possible impact of the core geometry is also not clear. Especially for mixed and orthogonal biasing, further investigations have to be made.

\section{Conclusion}

In the presented paper the impact of the implementation of the auxiliary bias-winding was studied for mixed biasing. Seven different implementation designs were investigated in small signal measurements and 3D-FEM-simulations. The results were proven by calculated results of equivalent diagram circuits, which have been developed.

For the standard crosswise current arrangement (Designs A to C) experimental and simulation results are similar. That leads to the conclusion that there is no impact of the implementation of the auxiliary bias-winding on the magnetic device. However, a reduced saturation flux density of the ferrite core with drill holes was noted.

In further investigations the arrangement of the auxiliary current was adapted in a horizontal current arrangement (Designs D and E). According to the crosswise arrangement, no impact of the implementation of the auxiliary bias-winding on the magnetic device could be detected. However, the effect of mixed biasing is mitigated using that arrangement.

The current arrangement was changed to a vertical auxiliary current arrangement (Design F and G). Due to an auxiliary magnetic flux which effects the whole magnetic core, the magnetization curve becomes unsymmetrical, resulting in a behavior like a "magnetic diode". Simulation results for both designs are different in relation to the implementation of the auxiliary bias-winding. The 
magnetization curve can be rotated by $180^{\circ}$ if Design $\mathrm{G}$ is used instead of Design $\mathrm{F}$ without changing the directions of main and auxiliary current.

The performed investigations, especially the calculations show that the magnetization curve of a ferrite core can be calculated by integrating the small signal measurement results. The prerequisite is that measurements are performed by using the "parallel equivalent circuit" setting of the measuring instrument.

This paper shows that there new possibilities of controlling magnetic devices by using drill holes within the magnetic core material. However, further investigations have to be made to develop an explanation.

\section{References}

[1] Dennis Eichhorst, Jonas Pfeiffer and Peter Zacharias. (2019, 05). Weight reduction of DC/DC converters using controllable inductors. Presented at PCIM Europe.

[2] Peter Zacharias, Thiemo Kleeb, Florian Fenske, Jiajing Wende and Jonas Pfeiffer. (2017, 09). Controlled magnetic devices in power electronic applications. Presented at EPE ECCE Europe.

[3] Jens Friebe, "Permanentmagnetische Vormagnetisierung von Speicherdrosseln in Stromrichtern," Ph.D. dissertation, Dept. of Elect. Eng., University of Kassel, Kassel, Germany, 2014.

[4] Dale S. L. Dolan, "Modelling and performance evaluation of the virtual air gap variable reactor," Ph.D. dissertation, Grad. Dept. of Elect. and Comp. Eng., University of Toronto, Toronto, Canada, 2009.

[5] V. Molcrette, Jean-Luc Kotny, J.-P. Swan and Jean-Francois Brudny. "Reduction of inrush current in singlephase transformer using virtual air gap technique," IEEE Transactions on Magnetics., vol. 34, no. 4, pp. 1192 1194, Jul. 1998.

[6] J. Avila-Montes and Enrique Melgoza. (2012, 09). Scaling the virtual air-gap principle to high voltage large power applications. Presented at ICEIMach.

[7] Enrique Melgoza, J. Avila-Montes and Manuel Madrigal. (2013, 11). Analysis of the magnetic characteristics of virtual-gap reactors. Presented at ROPEC.

[8] Adalbert Konrad and Jean-Francois Brudny. "Virtual air gap length computation with the finite-element method," IEEE Transactions on Magnetics., vol. 43, no. 4, pp. 1829-1832, Mar. 2007.

[9] Ali A. Abrishami and Hossein Heydari. (2014, 05). Improved accuracy for finite element modeling in virtual air gap lenght computation. Presented at EEEIC.

[10] Jean-Francois Brudny, Guillaume Parent and Ines Naceur. "Characterization and modeling of a virtual air gap by means of a reluctance network," IEEE Transactions on Magnetics., vol. 53, no. 7, sequence no. 8002007, Jul. 2017.

[11] S. Magdaleno and C. P. Rojas. (2010, 10). Control of the magnetizing characteristics of a toroidal core using virtual gap. Presented at CERMA.

[12] Carrol W. Lufcy, “A survey of magnetic amplifiers,” Proceedings of the IRE., vol. 43, no. 4, pp. 404-413, Apr. 1955.

[13] Marina S. Perdigao, Maikel Menke, Alysson R. Seidel, Rafael A. Pinto and Jose M. Alonso. (2014, 10). A review on variable inductors and variable transformers: Applications to lighting drivers. Presented at IEEE Industry Application Society Annual Meeting. 